Wenhui Luo*

\title{
Further extensions of Hartfiel's determinant inequality to multiple matrices
}

https://doi.org/10.1515/spma-2020-0125

Received December 17, 2020; accepted January 21, 2021

Abstract: Following the recent work of Zheng et al., in this paper, we first present a new extension Hartfiel's determinant inequality to multiple positive definite matrices, and then we extend the result to a larger class of matrices, namely, matrices whose numerical ranges are contained in a sector. Our result complements that of Mao.

Keywords: determinant inequality, positive definite matrix, numerical range, sector matrix

MSC: $15 \mathrm{~A} 45,15 \mathrm{~A} 60$

\section{Introduction}

Throughout the paper, we denote by $\mathbb{M}_{n}$ the set of $n \times n$ complex matrices. Recall that the numerical range (see, e.g., [3]) of $A \in \mathbb{M}_{n}$ is defined as the set on the complex plane

$$
W(A)=\left\{v^{*} A v: v \in \mathbb{C}^{n}, v^{*} v=1\right\} .
$$

For a fixed $\theta \in[0, \pi / 2)$, obviously the set on the complex plane

$$
S_{\theta}=\{z \in \mathbb{C}: \Re z>0,|\Im z| \leq(\Re z) \tan \theta\}
$$

is a sector excluding the vertex. We shall mainly consider matrices whose numerical range is contained in $S_{\theta}$, the so called sector matrices [11]. For any $A \in \mathbb{M}_{n}$, its real (or Hermitian) part is denoted by $\Re A:=\left(A+A^{*}\right) / 2$. Clearly, if $W(A) \subset S_{\theta}$, then $\Re A$ is positive definite.

A fundamental determinant inequality states that if $A, B \in \mathbb{M}_{n}$ are positive definite, then

$$
\operatorname{det}(A+B) \geq \operatorname{det} A+\operatorname{det} B .
$$

In [5], Haynsworth proved the following improvement of (1).

Theorem 1.1. [5, Theorem 3] Suppose $A, B \in \mathbb{M}_{n}$ are positive definite. Let $A_{k}$ and $B_{k}, k=1, \ldots, n-1$, denote the kth leading principal submatrices of $A$ and $B$, respectively. Then

$$
\operatorname{det}(A+B) \geq\left(1+\sum_{k=1}^{n-1} \frac{\operatorname{det} B_{k}}{\operatorname{det} A_{k}}\right) \operatorname{det} A+\left(1+\sum_{k=1}^{n-1} \frac{\operatorname{det} A_{k}}{\operatorname{det} B_{k}}\right) \operatorname{det} B .
$$

Using a clever argument, Hartfiel [4] refined Haynsworth's result by adding a nonnegative term on the right side of the inequality.

`Corresponding Author: Wenhui Luo: Department of Education and Educational Technology, Jiangmen Polytechnic, Jiangmen, Guangdong, China, E-mail: Lwh-822@163.com 
Theorem 1.2. [4] Under the same condition as in Theorem 1.1,

$$
\begin{aligned}
\operatorname{det}(A+B) \geq( & \left.1+\sum_{k=1}^{n-1} \frac{\operatorname{det} B_{k}}{\operatorname{det} A_{k}}\right) \operatorname{det} A+\left(1+\sum_{k=1}^{n-1} \frac{\operatorname{det} A_{k}}{\operatorname{det} B_{k}}\right) \operatorname{det} B \\
& +\left(2^{n}-2 n\right) \sqrt{\operatorname{det} A B} .
\end{aligned}
$$

Hartfiel's inequality (2) has been extended to sector matrices by a number of authors; see [8, 10, 14, 17]. In [8], Hou and Dong extended Hartfiel's inequality to a triple of matrices. By making use of Hou and Dong's result, Zheng et al. [17] improved and extended the main result in [10], moreover, they obtained the following two theorems.

Theorem 1.3. [17, Theorem 2.6] Suppose $A, B \in \mathbb{M}_{n}$ such that $W(A), W(B) \subset S_{\theta}$. Let $A_{k}$ and $B_{k}, k=1, \ldots, n-$ 1 , denote the kth leading principal submatrices of $A$ and $B$, respectively. Then

$$
\begin{aligned}
\sec ^{2 n-1}(\theta)|\operatorname{det}(A+B)| \geq & \left(1+\sum_{k=1}^{n-1}\left|\frac{\operatorname{det} B_{k}}{\operatorname{det} A_{k}}\right|\right)|\operatorname{det} A|+\left(1+\sum_{k=1}^{n-1}\left|\frac{\operatorname{det} A_{k}}{\operatorname{det} B_{k}}\right|\right)|\operatorname{det} B| \\
& +\left(2^{n}-2 n\right) \sqrt{|\operatorname{det} A B|} .
\end{aligned}
$$

Remark 1.4. In [10], Lin proved a weaker result with the first coefficient $\sec ^{3 n-2}(\theta)$ instead of $\sec ^{2 n-1}(\theta)$.

Theorem 1.5. [17, Theorem 2.8] Let $M, N, L \in \mathbb{M}_{n}$ such that $W(M), W(N), W(L) \subset S_{\theta}$. Then it holds

$$
\begin{aligned}
|\operatorname{det}(M+N+L)| \geq & \prod_{j=1}^{n}\left(\frac{\left|\operatorname{det} N_{j}\right|}{\left|\operatorname{det} N_{j-1}\right|}+\frac{\left|\operatorname{det} L_{j}\right|}{\left|\operatorname{det} L_{j-1}\right|}\right) \cos ^{j}(\theta) \\
& +\prod_{j=1}^{n}\left(\frac{\left|\operatorname{det} L_{j}\right|}{\left|\operatorname{det} L_{j-1}\right|}+\frac{\left|\operatorname{det} M_{j}\right|}{\left|\operatorname{det} M_{j-1}\right|}\right) \cos ^{j}(\theta) \\
& +\prod_{j=1}^{n}\left(\frac{\left|\operatorname{det} M_{j}\right|}{\left|\operatorname{det} M_{j-1}\right|}+\frac{\left|\operatorname{det} N_{j}\right|}{\left|\operatorname{det} N_{j-1}\right|}\right) \cos ^{j}(\theta) \\
& -(|\operatorname{det} M|+|\operatorname{det} N|+|\operatorname{det} L|),
\end{aligned}
$$

where by convention, $\operatorname{det} M_{0}=\operatorname{det} N_{0}=\operatorname{det} L_{0}=1$.

Very recently, Mao in [14] extended Theorem 1.3 to any number of sector matrices. More precisely, she obtained the following result.

Theorem 1.6. Let $A_{j} \in \mathbb{M}_{n}$ with $W\left(A_{j}\right) \subset S_{\theta}$, and let $A_{j k}, k=1, \ldots, n-1$, denote the $k$ th leading principal submatrix of $A_{j}, j \in \mathcal{M}:=\{1, \ldots, m\}$. Then

$$
\begin{aligned}
\sec ^{2 n-1}(\theta)\left|\operatorname{det}\left(\sum_{j=1}^{m} A_{j}\right)\right| \geq \sum_{j=1}^{m} & \left(1+\sum_{k=1}^{n-1} \frac{\sum_{\substack{i \in \mathcal{M} \\
i \neq j}}\left|\operatorname{det} A_{i k}\right|}{\left|\operatorname{det} A_{j k}\right|}\right)\left|\operatorname{det} A_{j}\right| \\
& +\left(2^{n}-2 n\right) \sum_{1 \leq i<j \leq m} \sqrt{\left|\operatorname{det} A_{i} A_{j}\right|}
\end{aligned}
$$

The main goal of the present paper is to extend Theorem 1.5 to any number of sector matrices. To this end, we first present a relevant result for positive definite matrices. Some corollaries are included.

\section{Main Results}

Before stating our results, we need to present some lemmas that are useful in our proofs. 
The first lemma is folklore in matrix analysis.

Lemma 2.1. [5, Lemma 2] Let $A, B \in \mathbb{M}_{n}$ be positive definite and let $A_{j}, B_{j}$ denote the kth leading principal submatrix of $A, B$, respectively. Then

$$
\frac{\operatorname{det}\left(A_{j}+B_{j}\right)}{\operatorname{det}\left(A_{j-1}+B_{j-1}\right)} \geq \frac{\operatorname{det} A_{j}}{\operatorname{det} A_{j-1}}+\frac{\operatorname{det} B_{j}}{\operatorname{det} B_{j-1}}, \quad j=1, \ldots, n,
$$

where by convention $\operatorname{det} A_{0}=\operatorname{det} B_{0}=0$.

The second lemma is known as the Ostrowski-Taussky inequality (see [7, p. 510]).

Lemma 2.2. Let $A \in \mathbb{M}_{n}$ with $\Re A$ positive definite. Then

$$
\operatorname{det}(\Re A) \leq|\operatorname{det} A| .
$$

The third lemma gives a reverse of the Ostrowski-Taussky inequality.

Lemma 2.3. [10, Lemma 2.6] Let $A \in \mathbb{M}_{n}$ with $W(A) \subset S_{\theta}$. Then

$$
\operatorname{det}(\Re A) \geq \cos ^{n}(\theta)|\operatorname{det} A| .
$$

The fourth lemma pays a key role in our new extension of Hartfiel's inequality to multiple positive definite matrices.

Lemma 2.4. (ref. [1, Corollary 4.4]) Suppose $A_{j} \in \mathbb{M}_{n}, j \in 1, \ldots, m$, are positive definite. Then

$$
\operatorname{det}\left(\sum_{j=1}^{m} A_{j}\right)+(m-2) \sum_{j=1}^{m} \operatorname{det} A_{j} \geq \sum_{1 \leq i<j \leq m} \operatorname{det}\left(A_{i}+A_{j}\right) .
$$

It is worthy to mention that in [1, Corollary 4.4], the authors stated their result for the generalized matrix functions, which includes the determinant as a special case. The inequality in Lemma 2.4 is a direct extension of the main result in [13].

Below is our extension of Hartfiel's inequality to multiple positive definite matrices.

Proposition 2.5. Let $A_{j} \in \mathbb{M}_{n}, j=1, \ldots, m$, be positive definite, and let $A_{j k}, k=1, \ldots, n-1$, denote the $k$ th leading principal submatrix of $A_{j}$. Then

$$
\operatorname{det}\left(\sum_{j=1}^{m} A_{j}\right) \geq \sum_{1 \leq i<j \leq m} \prod_{k=1}^{n}\left(\frac{\operatorname{det} A_{i k}}{\operatorname{det} A_{i(k-1)}}+\frac{\operatorname{det} A_{j k}}{\operatorname{det} A_{j(k-1)}}\right)-(m-2) \sum_{j=1}^{m} \operatorname{det} A_{j},
$$

where by convention det $A_{j 0}=1$ for all $j$.

Proof. Applying Lemma 2.1 to $A_{i}, A_{j}$ gives

$$
\frac{\operatorname{det}\left(A_{i k}+A_{j k}\right)}{\operatorname{det}\left(A_{i(k-1)}+A_{j(k-1)}\right)} \geq \frac{\operatorname{det} A_{i k}}{\operatorname{det} A_{i(k-1)}}+\frac{\operatorname{det} A_{j k}}{\operatorname{det} A_{j(k-1)}}, \quad j=1, \ldots, n .
$$

Taking products for $k$ from 1 to $n$ gives

$$
\operatorname{det}\left(A_{i}+A_{j}\right) \geq \prod_{k=1}^{n}\left(\frac{\operatorname{det} A_{i k}}{\operatorname{det} A_{i(k-1)}}+\frac{\operatorname{det} A_{j k}}{\operatorname{det} A_{j(k-1)}}\right) .
$$

Now by Lemma 2.4, we have

$$
\operatorname{det}\left(\sum_{j=1}^{m} A_{j}\right) \geq \sum_{1 \leq i<j \leq m} \operatorname{det}\left(A_{i}+A_{j}\right)-(m-2) \sum_{j=1}^{m} \operatorname{det} A_{j}
$$




$$
\geq \sum_{1 \leq i<j \leq m} \prod_{k=1}^{n}\left(\frac{\operatorname{det} A_{i k}}{\operatorname{det} A_{i(k-1)}}+\frac{\operatorname{det} A_{j k}}{\operatorname{det} A_{j(k-1)}}\right)-(m-2) \sum_{j=1}^{m} \operatorname{det} A_{j} .
$$

This completes the proof.

The following result extends Theorem 1.5.

Theorem 2.6. Let $A_{j} \in \mathbb{M}_{n}, j=1, \ldots, m$, such that $W\left(A_{j}\right) \subset S_{\theta}$, and let $A_{j k}, k=1, \ldots, n-1$, denote the $k$ th leading principal submatrix of $A_{j}$. Then it holds

$$
\left|\operatorname{det}\left(\sum_{j=1}^{m} A_{j}\right)\right| \geq \sum_{1 \leq i<j \leq m} \prod_{k=1}^{n}\left(\frac{\left|\operatorname{det} A_{i k}\right|}{\left|\operatorname{det} A_{i(k-1)}\right|}+\frac{\left|\operatorname{det} A_{j k}\right|}{\left|\operatorname{det} A_{j(k-1)}\right|}\right) \cos ^{k} \theta-(m-2) \sum_{j=1}^{m}\left|\operatorname{det} A_{j}\right|,
$$

where by convention $\operatorname{det} A_{j 0}=1$ for all $j$.

Proof. First of all, since $W\left(\sum_{j=1}^{m} A_{j}\right) \subset S_{\theta}$, then by Lemma 2.2,

$$
\begin{aligned}
\left|\operatorname{det}\left(\sum_{j=1}^{m} A_{j}\right)\right| & \geq \operatorname{det}\left(\Re \sum_{j=1}^{m} A_{j}\right) \\
& =\operatorname{det}\left(\sum_{j=1}^{m} \Re A_{j}\right) .
\end{aligned}
$$

As $\Re A_{j}$ are positive definite for all $j$, we can apply Proposition 2.5 to get

$$
\begin{aligned}
\left|\operatorname{det}\left(\sum_{j=1}^{m} A_{j}\right)\right| & \geq \sum_{1 \leq i<j \leq m} \prod_{k=1}^{n}\left(\frac{\operatorname{det} \Re A_{i k}}{\operatorname{det} \Re A_{i(k-1)}}+\frac{\operatorname{det} \Re A_{j k}}{\operatorname{det} \Re A_{j(k-1)}}\right)-(m-2) \sum_{j=1}^{m} \operatorname{det} \Re A_{j} \\
& \geq \sum_{1 \leq i<j \leq m} \prod_{k=1}^{n}\left(\frac{\operatorname{det} \Re A_{i k}}{\left|\operatorname{det} A_{i(k-1)}\right|}+\frac{\operatorname{det} \Re A_{j k}}{\left.\mid \operatorname{det} A_{j(k-1)}\right)}\right)-(m-2) \sum_{j=1}^{m}\left|\operatorname{det} A_{j}\right| \\
& \geq \sum_{1 \leq i<j \leq m} \prod_{k=1}^{n}\left(\frac{\left|\operatorname{det} A_{i k}\right|}{\left|\operatorname{det} A_{i(k-1)}\right|}+\frac{\left|\operatorname{det} A_{j k}\right|}{\left|\operatorname{det} A_{j(k-1)}\right|}\right) \cos ^{k} \theta-(m-2) \sum_{j=1}^{m}\left|\operatorname{det} A_{j}\right|,
\end{aligned}
$$

in which the second inequality is by Lemma 2.2 and the third inequality is by Lemma 2.3, respectively. This completes the proof.

A matrix $A \in \mathbb{M}_{n}$ is called accretive-dissipative if both real part $\Re A$ and imaginary part $\Im A:=\left(A-A^{*}\right) / 2 i$ (in the sense of Cartesian decomposition) are positive definite. This class of matrices has appeared in numerical linear algebra $[2,6,12]$ and has been studied recently by a number of authors $[9,15,16]$. Note that $M$ is accretive-dissipative if and only if $W\left(e^{-i \pi / 4} M\right) \subset \mathcal{S}_{\pi / 4}$. This observation enables us to state the following two corollaries.

Corollary 2.7. Let $A_{j} \in \mathbb{M}_{n}, j=1, \ldots, m$, be accretive-dissipative, and let $A_{j k}, k=1, \ldots, n-1$, denote the $k$ th leading principal submatrix of $A_{j}$. Then it holds

$$
\begin{aligned}
\left|\operatorname{det}\left(\sum_{j=1}^{m} A_{j}\right)\right| \geq & \sum_{1 \leq i<j \leq m} \prod_{k=1}^{n}\left(\frac{\left|\operatorname{det} A_{i k}\right|}{\left|\operatorname{det} A_{i(k-1)}\right|}+\frac{\left|\operatorname{det} A_{j k}\right|}{\left|\operatorname{det} A_{j(k-1)}\right|}\right) 2^{-k / 2} \\
& -(m-2) \sum_{j=1}^{m}\left|\operatorname{det} A_{j}\right|
\end{aligned}
$$

where by convention $\operatorname{det} A_{j 0}=1$ for all $j$. 
Proof. Since $A_{j} \in \mathbb{M}_{n}$ are accretive-dissipative, we have $W\left(e^{-i \pi / 4} A_{j}\right) \subset \mathcal{S}_{\pi / 4}$ for all $j$. Then we apply Theorem 2.6 to the matrices $e^{-i \pi / 4} A_{j}$ to get the result, because in this case $\cos ^{k} \theta=2^{-k / 2}$.

Acknowledgements: The author is grateful to Jiajie Xu for some helpful discussions.

Data Availability Statement: Data sharing is not applicable to this article as no datasets were generated or analyzed during the current study.

Competing interests: The author declares that he have no competing interests.

Funding: The research was supported in part by Jiangmen Polytechnic.

Authors' contributions: Wenhui Luo is the sole contributor to this work. He read and approved the final manuscript.

\section{References}

[1] W. Berndt, S. Sra, Hlawka-Popoviciu inequalities on positive definite tensors, Linear Algebra Appl. 486 (2015) 317-327.

[2] A. George, K.D. Ikramov, A.B. Kucherov, On the growth factor in gaussian elimination for generalized Higham matrices, Numer. Linear Algebra Appl. 9 (2002) 107-114.

[3] K. E. Gustafson, D. K. M. Rao, Numerical Range: The Field of Values of Linear Operators and Matrices, Springer, New York, 1997.

[4] D. J. Hartfiel, An extension of Haynsworth's determinant inequality, Proc. Amer. Math. Soc. 41 (1973) 463-465.

[5] E. V. Haynsworth, Applications of an inequality for the Schur complement, Proc. Amer. Math. Soc. 24 (1970) 512-516.

[6] N.J. Higham, Factorizing complex symmetric matrices with positive real and imaginary parts, Math. Comput. 67 (1998) 15911599.

[7] R.A. Horn and C.R. Johnson, Matrix Analysis, Cambridge University Press, Cambridge, 2013.

[8] L. Hou, S. Dong, An extension of Hartfiel's deteminant inequality, Math. Inequal. \& Appl. 21 (2018) 1105-1110.

[9] M. Lin, Fischer type determinantal inequalities for accretive-dissipative matrices. Linear Algebra Appl. 438 (2013) $2808-2812$.

[10] M. Lin, Extension of a result of Hanynsworth and Hartfiel, Arch. Math. 1 (2015) 93-100.

[11] M. Lin, Some inequalities for sector matrices, Oper. Matrices, 10 (2016) 915-921.

[12] M. Lin, A note on the growth factor in gaussian elimination for accretive-dissipative matrices, Calcolo 51 (2014) 363-366.

[13] M. Lin, A determinantal inequality for positive definite matrices, Electron J. Linear Algebra 27 (2014), 821-826.

[14] Y. Mao, Extensions of Hartfiel's inequality to multiple matrices, Linear Algebra Appl 589 (2020) 96-102.

[15] J. Xue, X. Hu, On Fischer-type determinantal inequalities for accretive-dissipative matrices, Journal of Inequalities and Applications (2015) 2015:194.

[16] J. Yang, Some determinantal inequalities for accretive-dissipative matrices. J. Inequal. Appl. (2013)2013, 512.

[17] Y. Zheng, X. Jiang, X. Chen, F. Alsaadi, More extensions of a determinant inequality of Hartfiel, Applied Math. Comput. 369 (2020) 124827. 